\title{
Dynamic Reactive Power Optimization Based on Genetic Algorithms in Power Systems
}

\author{
Ming Zhu \\ Benxi Power Supply Branch \\ State Grid Liaoning Electric Power Supply Co. Ltd \\ Benxi, China \\ e-mail: bxwl_650928@126.com
}

Zhida Xu

Benxi Power Supply Branch

State Grid Liaoning Electric Power Supply Co. Ltd Benxi, China

e-mail: bxwl_650928@126.com

\author{
Xuebin Li \\ Benxi Power Supply Branch \\ State Grid Liaoning Electric Power Supply Co. Ltd \\ Benxi, China \\ e-mail: lixb@sohu.com \\ Chengming Jin \\ Information and Communication Branch \\ State Grid Liaoning Electric Power Supply Co. Ltd \\ Benxi, China \\ e-mail: jcm@ln.sgcc.com.cn
}

\begin{abstract}
The genetic algorithm is applied to reactive power optimization. The establishment of a genetic algorithm for reactive power optimization model based clustering algorithm for the day through the system load curve cluster sub-period, and in the establishment of dynamic reactive power optimization model type variety, based on the use of genetic algorithms in all the major periods of discrete control variables to optimize the adjustment. It successfully resolves the problem of reactive power optimization of discrete variable and avoids the problem of local optimum conventional mathematical optimization methods. In the meantime, the optimization given for reactive power optimization provides a new algorithm this algorithm, which has been used in IEEE 30bus system to improve the voltage quality of the network and reduce system power loss. Furthermore, it enhances the security, stability and economy of the entire grid, and achieves good results.
\end{abstract}

Keywords-power systems; genetic algorithm; dynamic reactive power optimization; IEEE 30-bus system

\section{INTRODUCTION}

Important measure both reactive power optimization of power system to ensure the security, stability and economic operation of an effective means, but also effectively reduce the power system network losses, improve voltage quality. Therefore, the power system power optimization study of the problem, therefore, in order to increase the voltage levels and reduce network losses as the main target of reactive power optimization research, both theoretical significance, but also has practical value, has been the subject of attention many relationships and non-linear search using sensitivity methods (such as: linear programming, nonlinear programming, mixed integer programming, sensitivity analysis, expert system model, interior point methods, multi-objective fuzzy mathematical programming) has been able to successfully resolve the power system power Optimization problems [1-4]. However, these methods have limitations in three aspects: First, the optimization of these methods are starting from a point along the search, so the results related to the selected initial point, if the initial point selected properly, will converge to a local optimum rather than the global optimal; Second performance indicators and constraints is difficult to fully reflect the system characteristics and can not guarantee the performance indicator is a convex function which makes the algorithm itself is difficult to give a global optimal solution, tend to converge to a local optimal solution; third is the corresponding optimization algorithms tend to be difficult to achieve dimension restrictions practical purposes.

Genetic algorithms (GA) as a new way to simulate the process of biological evolution, with its nonlinear and complex problems of global search capability and simple generic and robust notable feature, causing different research areas widespread attention [5]. Genetic algorithm is used stochastic optimization techniques, handling discrete variables through genetic manipulation, in order to obtain a greater probability of global optimal solution, while providing optimal range for practical engineering problems, suboptimal solutions to choose, to avoid dimension disaster, to meet the actual needs [6-9]. based on the genetic algorithm for dynamic reactive power optimization features and system load changes, the study of a class of intelligent power system dynamic reactive power optimization problem. According restraint system running economic targets and control equipment and other specific circumstances, the use of the system throughout the day clustering algorithm for clustering load curves subperiod, and then establish the basis of dynamic reactive power optimization model, the use of genetic algorithms for each big time discrete control variables to optimize the adjustment, to further improve the accuracy of the system optimization, the final day of the formation of dynamic reactive power optimization control strategy. Through the IEEE-30 bus system simulation analysis, the results of this paper show that intelligent dynamic reactive power optimization method can meet in case of system constraints, and effectively improve network voltage quality and reduce power loss and achieve security, stability and economic systems operation. 


\section{Genetic Algorithm In REACtive Power OPTIMIZATION}

Based on Genetic Algorithm reactive power optimization problem solving, first control variable chromosome coding, individuals within a certain range of the formation of a certain number of randomly generated population, then flow calculation made by the objective function value that each individual fitness value, according to adapt the size of the value of the selection operation, and then through crossover and mutation to produce new population control variables individuals, repeated iterations until convergence conditions are satisfied, get a group of optimal solution [10]. In the reactive power optimization problem, the specific operation on genetic algorithm is as follows:

\section{A. The individual Encoding}

Genetic algorithm is the basis of the individual encoding genetic manipulation. Reactive power optimization of power system primary control variable is the terminal voltage of the generator, transformer load tap ratio and switching shunt capacitors. Which OLTC transformer turns ratio is adjusted according to the file, but also by groups shunt capacitor switching, is a discrete variable, the terminal voltage of the generator can be continuously smooth adjustment, is a continuous variable. In this paper the genetic algorithm is mainly used to optimize the value of reactive power optimization problem discrete control variables, so the use of binary encoding of individuals, each variable length encoding takes 10 binary number. The dimension of the population of individuals for the number of control variables, which can be coded as follows:

$$
X=\left[T_{t 1}, T_{t 2}, \cdots, T_{t j}\left|Q_{C 1}, Q_{C 2}, \cdots, Q_{C k}\right| U_{G 1}, U_{G 2}, \cdots, U_{G i}\right]
$$

Where $T_{t}$ is OLTC transformer ratio corresponding encoded string, $Q_{C}$ is the capacity of reactive power compensation capacitors in parallel corresponding coding sequence, $U_{G}$ is the terminal voltage corresponding coding sequence generator.

\section{B. Design fitness function}

Genetic Algorithms in Search to fitness function is based, does not rely on external information. Fitness function based objective function transformed from the reactive power optimization through flow calculation can be drawn from the system active power loss and variable state to obtain the objective function value. But no power optimization objective function for obtaining the minimum value problems, genetic algorithm, the fitness function is usually to strike a great value, the greater the fitness value, the more outstanding individuals, was chosen to copy the greater the probability. Therefore, the objective function needs to be converted to the appropriate fitness function, this paper chooses to take reciprocal approach, the objective function is $F$, then the fitness function is:

$$
f=1 / F
$$

\section{Genetic Operators operating}

Genetic operators is a key step in genetic algorithm. Select Options and individual size of fitness value, according to the proportion of individuals choose to copy the fitness values. In this paper, roulette way to achieve, first calculate the value of each individual's fitness, the fitness value of this share of the population fitness probability is the ratio of the sum of the values of the individual selected. Population size set for $n$, the individual $i$ of the fitness value set for $f_{i}$, then the individual $i$ probability of being set for:

$$
p_{i}=f_{i} / \sum_{i=1}^{n} f_{i} \quad i=1,2, \cdots, n
$$

Cross-operation is an important process to generate new individuals, this paper uses two-point crossover crossover operation, first to the population in each of the two individuals were paired for a group, and then generate a random number between 0 and 1 , and the set of crossover probability comparison, when the random number is less than the probability of individual variables crossover double-point crossover operation that randomly generates two crossover points, the part of the intersection between the two individual variables were interchanged to produce new individuals. Two-point crossover model with a strong reorganization ability to overcome some modes deceptive and breakthroughs local extreme points.

Mutation process can prevent the loss of some useful genetic factors, maintaining the diversity of population. Under normal circumstances change mutation probability is small, the paper take a single point mutation, for the individual bits of string, when the random number generator is less than a given mutation probability negated bit of string operation, that bit of the original string is 0 to 1 , the original 1 is changed to 0 , thereby generating a new individual.

\section{Determine population size}

Individual populations composed by the control variables, namely the total population size of individuals in the population, too much or too little inappropriate total population of individuals, individual optimization algorithm is too poor, too many individual high computational complexity, increase the amount of computation and algorithmic search time, generally based on historical experience and specific questions selected population size, the paper size selected counted 30 cases of genetic algorithm combined with reactive power optimization based on experience.

\section{E. Convergence criterion set}

The genetic algorithm ensure maximum calculated globally optimal solution, avoid falling into local optimum, usually have to guarantee a certain number of iterations. But sometimes there is no maximum number of iterations before the emergence of the optimal solution has been reached, then the iteration is wasted computing time, then you can jump out of the iteration, the end of the operation, accelerate the optimization of search speed. In this paper, 
the maximum number of iterations and individual genetic fitness value to maintain optimal convergence criterion does not disguise a certain number of iterations based on a combination of specific problems, if the individual optimal fitness value after a certain number of iterations remains unchanged, then the end of the iteration optimal output solution, or reaches a predetermined number of iterations, the output of the search to obtain optimal solution. In this paper, examples of genetic algorithm to select the maximum number of iterations is 200 times.

\section{BASED ON GENETIC ALGORITHM OPTIMIZATION OF DYNAMIC REACTIVE POWER}

According to the characteristics of dynamic reactive power optimization, we use the method to divide the load curve on the grid to handle periods of reactive power control equipment bound action times, the 24-hour load in accordance with certain principles are divided into several large periods, the use of genetic algorithm for discrete control variable control strategy to strike on all the major periods, on the basis of various small-day period of continuous control variables using improved particle swarm optimization algorithm to optimize the adjustment, make full use of genetic algorithm and particle swarm optimization process variable characteristics, get the day's reactive power optimization control program.

\section{A. Mathematical model of dynamic reactive power optimization}

Dynamic reactive power optimization mathematical model taking into account the period of time (in this article is set to one day) of load change state to a minimum total active power loss within each period as the objective function, select the generator terminal voltage, load tap transformer ratio and reactive power compensation capacity switching for the control variables, the load node voltage and generator reactive power as state variables, the power constraint equation for the trend equations, variables, constraints include the control variables and state variables and load tap and adjust the number of capacitors in parallel constraint, the constraint of the state variables to be treated as a penalty function, Where $\Delta U_{\mathrm{i}}$ and $\Delta Q_{\mathrm{i}}$ according to the following formula values:

$$
\begin{gathered}
\Delta U_{\mathrm{i}}=\left\{\begin{array}{cc}
U_{i}-U_{i \max } & U_{i}>U_{i \max } \\
0 & U_{i \min } \leq U_{i} \leq U_{i \max } \\
U_{i \min }-U_{i} & U_{i}<U_{i \min }
\end{array}\right. \\
\Delta Q_{j}=\left\{\begin{array}{cc}
Q_{j}-Q_{j \max } & Q_{j}>Q_{j \max } \\
0 & Q_{j \min } \leq Q_{j} \leq Q_{j \max } \\
Q_{j \min }-Q_{j} & Q_{j}<Q_{j \min }
\end{array}\right.
\end{gathered}
$$

Where $M$ is the number of segments load, $P_{\text {kloss }}$ for system power loss, $N_{L}$ is the number of nodes load, $N_{G}$ is the generator nodes, $\lambda_{1}$ load node voltage bounds penalty coefficient, $\lambda_{2}$ is the generator reactive power output node bounds penalties coefficient, $U_{i}, U_{i \max }$ and $U_{i \text { min }}$ is the upper limit and lower limit for voltage load nodes and node voltage, $Q_{i} 、 Q_{i \max }$ and $Q_{i \text { min }}$ is the generator reactive power nodes, the power limit and lower limit. Constraint is:

$$
\begin{gathered}
\left\{\begin{array}{l}
P_{i}=U_{i} \sum_{j=1}^{n} U_{j}\left(G_{i j} \cos \delta_{i j}+B_{i j} \sin \delta_{i j}\right) \\
Q_{i}=U_{i} \sum_{j=1}^{n} U_{j}\left(G_{i j} \sin \delta_{i j}-B_{i j} \cos \delta_{i j}\right)
\end{array}\right. \\
\left\{\begin{array}{c}
U_{G i, \min } \leq U_{G i} \leq U_{G i, \max } i \in N_{G} \\
T_{t j, \min } \leq T_{t j} \leq T_{t j, \max } \quad j \in N_{T} \\
Q_{C k, \text { min }} \leq Q_{C k} \leq Q_{C k, \max } k \in N_{C} \\
Q_{G i, \min } \leq Q_{G i} \leq Q_{G i, \max } i \in N_{G} \\
U_{D j, \min } \leq U_{D j} \leq U_{D j, \max } j \in N_{D}
\end{array}\right.
\end{gathered}
$$

(6) and (7) is the static reactive power optimization constraints in the power constraint equation. (8) is the control device to adjust the number of constraints action, $S_{T \max }$ is within a day's maximum load tap adjust the number, $S_{C \max }$ is the greatest day to adjust the number of capacitors in parallel.

\section{B. Load curve segments}

The system clustering algorithm approach to the typical daily load curve, based on the load is divided into five big day period, then the system load curve segments were shown in Fig .1. 24 small load time is divided into five major periods, after staging a large load of each period is the average of the preceding segment load.

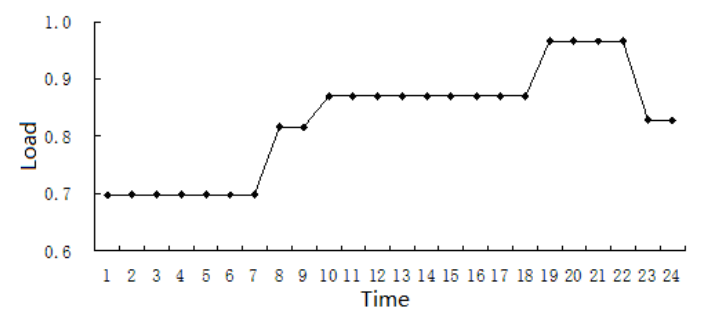

Figure 1. Daily load curve after segmenting 


\section{Cases and Analysis of Results}

Suppose OLTC transformer turns ratio and shunt capacitor switching times of the day does not exceed five times the maximum adjustment and network system load changes each node consistent trend, both in the load curve trends prevail, in order to IEEE-30 standard bus system for a given load value, the formation of dynamic reactive power optimization standard IEEE-30 node system.

The system clustering algorithm method, the 24-hour load curves divide large periods, according to the characteristics of the load curve, as early as during the evening peak to peak load change is not very dramatic, load curve can be divided into four sections, big-time load is average for the entire period of the load, shown in Fig .2.

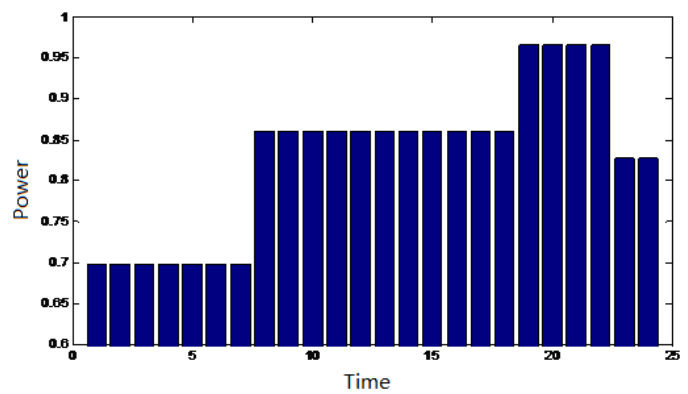

Figure 2. The curve of the daily load after segmenting

The compare of the proposed algorithm using optimization results and initial network losses as shown in TABLE I.

TABLE I. THE COMPARISON OF THE VARIOUS NeTwORK LOSSES

\begin{tabular}{|c|c|c|c|}
\hline Time & $\begin{array}{c}\text { Load } \\
\text { forecasting }\end{array}$ & $\begin{array}{c}\text { The initial net } \\
\text { loss }\end{array}$ & $\begin{array}{c}\text { Genetic } \\
\text { Algorithm }\end{array}$ \\
\hline 1 & 0.7258 & 0.04315 & 0.03725 \\
\hline 2 & 0.7014 & 0.04014 & 0.03328 \\
\hline 3 & 0.6819 & 0.03791 & 0.03259 \\
\hline 4 & 0.6779 & 0.03744 & 0.03185 \\
\hline 5 & 0.6872 & 0.03852 & 0.03286 \\
\hline 6 & 0.7105 & 0.04128 & 0.03573 \\
\hline 7 & 0.7065 & 0.04053 & 0.03373 \\
\hline 8 & 0.7914 & 0.05166 & 0.04238 \\
\hline 9 & 0.8394 & 0.05843 & 0.04837 \\
\hline 10 & 0.8781 & 0.06422 & 0.05236 \\
\hline 11 & 0.9010 & 0.06778 & 0.05481 \\
\hline 12 & 0.8123 & 0.05457 & 0.04454 \\
\hline 13 & 0.8203 & 0.05569 & 0.04596 \\
\hline 14 & 0.8577 & 0.06113 & 0.05066 \\
\hline 15 & 0.8768 & 0.06403 & 0.05160 \\
\hline 16 & 0.8917 & 0.06632 & 0.05439 \\
\hline 17 & 0.9052 & 0.06849 & 0.05542 \\
\hline 18 & 0.8852 & 0.06533 & 0.05329 \\
\hline 19 & 0.9331 & 0.07079 & 0.05783 \\
\hline 20 & 1.0000 & 0.08448 & 0.06840 \\
\hline 21 & 0.9846 & 0.08176 & 0.06719 \\
\hline 22 & 0.9455 & 0.07507 & 0.06235 \\
\hline 23 & 0.8601 & 0.06148 & 0.05103 \\
\hline 24 & 0.7951 & 0.05216 & 0.04283 \\
\hline
\end{tabular}

More than ten times the simulation results are averaged to get running, it can be seen by comparing the above results, the optimization of the system before the day of the total active power loss for $1.3824 \mathrm{pu}$, after a day of using genetic algorithms to optimize the total active power loss is $1.1407 \mathrm{pu}$, net loss decreased rate of $17.48 \%$, active power loss system has been greatly reduced, the voltage level stable within a certain range, and no more limited circumstances, the economy and security of the system has been greatly improved.

\section{CONCLUSION}

Use this method to get the control scheme, the control device in the number of full days of action has been effectively controlled, are within the acceptable range, improving the life of the equipment, but also reduce the work intensity of the operator. The simulation results and analysis can be obtained, the proposed method of dynamic reactive power optimization based on genetic algorithms can meet under controlled conditions in a number of operations constrained devices, improve voltage quality of the network, reduce system power loss, enhance the security of the entire grid, stability and economy.

\section{ACKNOWLEDGMENT}

The authors gratefully acknowledge the contribution of co-workers and reviewers' comments.

\section{REFERENCES}

[1] H. Z. Cheng and P. H. Liao, "Optimal placement planning of compensating capacitors in distribution power sys-tems," Electric Power System Automation, vol. 11, pp. 3-11, Dec. 1987.

[2] Cuello-Reyna AA and Cedeno-Maldonado JR, "A differential evolution approach to optimal reactive power planning," IEEE/PES Transmission \& Distribution Conference and Exposition, vol. 29, pp. 1-7, Jan. 2006.

[3] Y. H. PU and Y. LI, "An Impoved Genetic Algorithm for Reactive Power Optimization,"Proceedings of the 30th Chinese Control Conference, pp. 2105-2109, Dec. 2011.

[4] D. Q. Can, Z. H. Qu and H. Z. Cai, " Large Scale VAR Optimization and Planning by Tabu Search,"Electric Power Systems Research, vol. 39, pp. 195-204, Feb. 1996.

[5] D. E. Goldberg, "Genetie Algorithms in Seareh Optimization and Machine Learnning," New York: Addison-Wesley, 1989.

[6] K. Nara, "Implementatin of genetic algorithm for distribution systems loss minimum reconfiguration,"IEEE Trans PWRS, , vol. 314, pp. 1044-1051, Dec. 1992.

[7] M. Youshim, "Optimal economic power dispatch using genetic algorithms,"3rd ANNPS Japan,1993,pp. 157-162.

[8] K. Iba, "Reactive power optimization by genetic algorithm," IEEE Trans on Power Systems, vol. 9, pp. 685-692, Apr. 1994.

[9] K. Iba, “Optimal VAR allocation by genetic algorithm,"Proceeding of 1993 IEEE ANNPS' 93,Yokoyama, Japan,1993,pp. 163-168.

[10] S. Q. Song, H. D. Wang, F. H. Ban and C. Q. Yu, "Design and implementation of remote electric powermeter supervisory system,'RELAY, vol. 33, pp. 37-40, Nov. 2005. 